\title{
ac Josephson effect in the resonant tunneling through mesoscopic superconducting junctions
}

\author{
Yu Zhu ${ }^{1}$, Qing-feng Sun ${ }^{2}$, Wei $\mathrm{Li}^{1}$, Zhong-shui $\mathrm{Ma}^{1}$, and Tsung-han Lin ${ }^{1 *}$ \\ ${ }^{1}$ State Key Laboratory for Mesoscopic Physics and \\ Department of Physics, Peking University, Beijing \\ 100871, China \\ ${ }^{2}$ Center for the Physics of Materials and \\ Department of Physics, McGill University, Montreal, \\ PQ, Canada H3A 2T8
}

\begin{abstract}
We investigate ac Josephson effect in the resonant tunneling through mesoscopic superconducting junctions. In the presence of microwave irradiation, we show that the trajectory of multiple Andreev reflections can be closed by emitting or absorbing photons. Consequently, photon-assisted Andreev states are formed and play the role of carrying supercurrent. On the Shapiro steps, dc component appears when the resonant level $E_{0}$ is near the position $V_{L}+\frac{1}{2} m_{1} \omega=V_{R}+\frac{1}{2} m_{2} \omega(e=\hbar=1)$, where $m_{1}$ and $m_{2}$ are integers, $\omega$ is the frequency of microwave, $V_{L}$ and $V_{R}$ the chemical potentials of the superconducting leads. Analytical result is derived in the limit $\Delta \rightarrow \infty$, based on which new features of ac Josephson effect are revealed.

PACS numbers: 74.50.+r, 73.63.Kv, 73.23.Ad
\end{abstract}


Introduction-Since the achievement of atom-size superconducting quantum point contact (SQPC) by break junction technique [1-4], considerable theoretical works have been devoted to the transport problem in S-I-S or S-N-S structures ( $\mathrm{S}=$ superconductor, I=tunnel barrier, $\mathrm{N}=$ normal metal). Comparison between experimental data and theoretical calculation suggests that the coherent picture of multiple Andreev reflection (MAR) is the central conception in understanding the transport through such mesoscopic SQPC. Nevertheless, less attention is paid to the resonant tunneling (especially in ac transport) through mesoscopic superconducting junctions, in which the central region is consist of discrete electronic states. Such resonant tunneling can be achieved in nanoparticles sandwiched between superconducting membranes [5], or metallic clusters absorbed on the STM substrate [6], or a piece of carbon nanotube tunnel coupled to superconducting electrodes [7], or quantum dots fabricated in the S/2DEG hybrid structures [8], etc.

The transport through these structures is greatly modified due to the existence of discrete energy levels in the central region. It is shown by Yeyati et al. [9] and Johansson et al. [10] that the combination of MAR processes and resonant transmission gives rise to a rich subharmonic gap structure (SGS). Let us consider the transport through a resonant level with width $\Gamma$ coupled to $S$ leads with superconducting gap $\Delta$. In the limit $\Gamma \gg \Delta$, the resonance is sufficient broadened such that the I-V characteristics mimic that of SQPC. In the regime $\Gamma \sim \Delta$, pronounced SGS appears with concomitant negative differential conductance, which is distinguished from the nonresonant transport through SQPC. In the limit $\Gamma \ll$ $\Delta$, although SGS is more particular in the log-scale plot, the tunnel current as a whole is exponentially small. This is because a narrow resonance can not cover all the MAR trajectory, and it is very unlikely that multiple discrete levels are just in the position where MAR trajectory passes (see Fig.1a).

In this paper, we address the effect of microwave (MW) irradiation on the resonant tunneling through mesoscopic superconducting junctions. We show that the tunnel current is greatly enhanced on the Shapiro steps, when the resonant level $E_{0}$ is near the position $V_{L}+\frac{1}{2} m_{1} \omega=V_{R}+\frac{1}{2} m_{2} \omega$ ( $e=\hbar=1$ throughout), where $m_{1}$ and $m_{2}$ are integers, $\omega$ is the frequency of MW, $V_{L}$ and $V_{R}$ are the chemical potentials of left and right $\mathrm{S}$ lead. This can be attributed to the formation of photon-assisted Andreev states (PAAS), which play the role of carrying supercurrent. In the limit $\Delta \rightarrow \infty$, analytical results are derived, revealing new features of ac Josephson effect in the case of resonant tunneling.

Hamiltonian and Formulation-The model Hamiltonian reads $H=\sum_{\beta=L, R} H_{\beta}+H_{\text {cent }}+$ 
$H_{T}$, in which $H_{\beta}=\sum_{k \sigma} \varepsilon_{k} a_{\beta k \sigma}^{\dagger} a_{\beta k \sigma}+\sum_{k}\left(\Delta e^{\mathrm{i} \phi_{\beta}} a_{\beta k \uparrow}^{\dagger} a_{\beta-k \downarrow}^{\dagger}+H . c.\right)$ is the Hamiltonian for the $\beta$ th $\mathrm{S}$ lead, $H_{\text {cent }}=E_{0} \sum_{\sigma} c_{\sigma}^{\dagger} c_{\sigma}$ the resonant level in the central region, and $H_{T}=$ $\sum_{\beta k \sigma}\left(v_{\beta} \exp \left[\mathrm{i} \int_{0}^{t} \tilde{V}_{\beta}\left(t^{\prime}\right) d t^{\prime}\right] a_{\beta k \sigma}^{\dagger} c_{\sigma}+\right.$ H.c. $)$ the tunnel couplings. $\tilde{V}_{\beta}(t) \equiv V_{\beta}+W_{\beta} \cos \omega t$ is the time-dependent voltage drop across the $\beta$ th barrier, where $V_{\beta}$ is the chemical potential controlled by dc bias voltage and $W_{\beta} \cos \omega t$ the ac voltage induced by MW irradiation. For simplicity, we assume that Coulomb interaction and multi-level effect can be ignored in the central region. This assumption is somehow too ideal to achieve, but allows us to obtain analytical results and therefore instructive for the understanding of more complicated cases.

Keldysh Green functions are defined in the $2 \times 2$ Nambu representation:

$$
G_{t_{1} t_{2}}^{r, a,} \equiv\left(\begin{array}{cc}
\left\langle\left\langle c_{\uparrow}\left(t_{1}\right) \mid c_{\uparrow}^{\dagger}\left(t_{2}\right)\right\rangle\right\rangle^{r, a,<} & \left\langle\left\langle c_{\uparrow}\left(t_{1}\right) \mid c_{\downarrow}\left(t_{2}\right)\right\rangle\right\rangle^{r, a,<} \\
\left\langle\left\langle c_{\downarrow}^{\dagger}\left(t_{1}\right) \mid c_{\uparrow}^{\dagger}\left(t_{2}\right)\right\rangle\right\rangle^{r, a,<} & \left\langle\left\langle c_{\downarrow}^{\dagger}\left(t_{1}\right) \mid c_{\downarrow}\left(t_{2}\right)\right\rangle\right\rangle^{r, a,<}
\end{array}\right),
$$

The time-dependent current flowing out of the $\beta$ th lead can be expressed as

$$
I_{\beta}(t)=2 \operatorname{Re} \operatorname{Tr} \sigma_{z}\left(G^{r} \circ \Sigma_{\beta}^{<}+G^{<} \circ \Sigma_{\beta}^{a}\right)_{t t},
$$

in which $\sigma_{z}$ is the 3rd Pauli matrix, o the shorthand notation for integration over intermediate time variable, and $\Sigma_{\beta}$ the self energy due to tunnel coupling between the central region and the $\beta$ th lead. $G^{r}, G^{a}$, and $G^{<}$satisfy the integral equation:

$$
\begin{aligned}
G^{r, a} & =g^{r, a}+g^{r, a} \circ \Sigma^{r, a} \circ G^{r, a}, \\
G^{<} & =G^{r} \circ \Sigma^{<} \circ G^{a},
\end{aligned}
$$

in which

$$
\begin{aligned}
g_{t_{1} t_{2}}^{r, a} & =\int \frac{d \epsilon}{2 \pi} e^{-\mathrm{i} \epsilon\left(t_{1}-t_{2}\right)} g^{r, a}(\epsilon), \\
\Sigma_{\beta, t_{1} t_{2}}^{r, a,<} & =U_{\beta}\left(t_{1}\right) \int \frac{d \epsilon}{2 \pi} e^{-\mathrm{i} \epsilon\left(t_{1}-t_{2}\right)} \Sigma_{\beta}^{r, a,<}(\epsilon) U_{\beta}^{\dagger}\left(t_{2}\right),
\end{aligned}
$$

where

$$
\begin{aligned}
& g^{r, a}(\epsilon)=\left(\begin{array}{cc}
\frac{1}{\epsilon-E_{0} \pm \mathrm{i} 0^{+}} & 0 \\
0 & \frac{1}{\epsilon+E_{0} \pm \mathrm{i}^{+}}
\end{array}\right), \\
& \Sigma_{\beta}^{r, a}(\epsilon)=\mp \frac{\mathrm{i}}{2} \Gamma_{\beta} \frac{\epsilon \pm \mathrm{i} \eta}{\sqrt{(\epsilon \pm \mathrm{i} \eta)^{2}-\Delta^{2}}}\left(\begin{array}{cc}
1 & -\frac{\Delta}{\epsilon \pm i \eta} e^{-\mathrm{i} \phi_{\beta}} \\
-\frac{\Delta}{\epsilon \pm i \eta} e^{+\mathrm{i} \phi_{\beta}} & 1
\end{array}\right),
\end{aligned}
$$




$$
\begin{aligned}
& \Sigma_{\beta}^{<}(\epsilon)=f(\epsilon)\left[\Sigma_{\beta}^{a}(\epsilon)-\Sigma_{\beta}^{r}(\epsilon)\right], \\
& U_{\beta}(t)=\left(\begin{array}{cc}
\exp \left[-\mathrm{i} \int_{0}^{t} \tilde{V}_{\beta}\left(t^{\prime}\right) d t^{\prime}\right] & 0 \\
0 & \exp \left[+\mathrm{i} \int_{0}^{t} \tilde{V}_{\beta}\left(t^{\prime}\right) d t^{\prime}\right]
\end{array}\right),
\end{aligned}
$$

with $\Gamma_{\beta}$ being the coupling strength, $\eta$ the dephasing rate in the $\mathrm{S}$ lead, $f(\epsilon)$ the Fermi distribution function, and $\operatorname{Im} \sqrt{z}>0$ as a convention. The remaining task is to solve these integral equations and evaluate the dc component of the time-dependent current.

There are two intrinsic frequencies in the problem, $\omega_{1}=2 V=2\left(V_{L}-V_{R}\right)$ and $\omega_{2}=\omega$. Generally, one may perform a Fourier transform

$$
A_{t_{1} t_{2}}=\sum_{l_{1} l_{2}} e^{\mathrm{i}\left(l_{1} \omega_{1}+l_{2} \omega_{2}\right) t_{1}} \int \frac{d \epsilon}{2 \pi} e^{-\mathrm{i} \epsilon\left(t_{1}-t_{2}\right)} \tilde{A}_{l_{1} l_{2}}(\epsilon),
$$

and derive the recursive relation for $\tilde{A}_{l_{1} l_{2}}(\epsilon)$, as done in [11]. The calculation in this way relies on the numerical computing power, and analytical results are impossible. We note, however, that in the case of narrow resonance, dc component appears only if $\omega_{1}=N \omega_{2}$ with $N$ being an integer. This becomes clear by considering PAAS shown in Fig.1b and 1c: an electron (a hole) incomes through the resonant level $E_{0}$ and Andreev reflected by the right superconducting gap, the reflected hole (the reflected electron) exchanges $m_{1}$ photons with the MW field so that it can again passes through $E_{0}$. Then the hole (the electron) is Andreev reflected by the right superconducting gap as an electron (a hole), and exchanges $m_{2}$ photons to close the trajectory (detailed discussion for photon-assisted Andreev reflection is available in [12]). It is easy to see that the formation of PAAS requires $2 V=\left(m_{1}+m_{2}\right) \omega$. For this reason, we shall only consider the case of $V=V_{N}=\frac{N}{2} \omega$, while the current deviated from this condition is negligible small.

The problem is largely simplified since $\omega$ can be used as the basic frequency in the Fourier expansion. Define the Fourier transformation as

$$
\begin{aligned}
A_{t_{1} t_{2}} & =\sum_{l} e^{\mathrm{i} l \omega t_{1}} \int \frac{d \epsilon}{2 \pi} e^{-\mathrm{i} \epsilon\left(t_{1}-t_{2}\right)} \tilde{A}_{l}(\epsilon), \\
\mathbf{A}_{m n}(\epsilon) & =\tilde{A}_{m-n}(\epsilon-n \omega) .
\end{aligned}
$$

The definition guarantees the nice property that if $C=A \circ B$ then $\mathbf{C}_{m n}(\epsilon)=$ $\sum_{k} \mathbf{A}_{m k}(\epsilon) \mathbf{B}_{k n}(\epsilon)$. The Fourier transformed $g^{r, a}$ and $\Sigma_{\beta}^{r, a,<}$ are

$$
\mathbf{g}_{m n}^{r, a}(\epsilon)=\delta_{m n} g^{r, a}(\epsilon-n \omega)
$$




$$
\begin{aligned}
& \Sigma_{R, m n}^{r, a,<}(\epsilon)=\sum_{l}\left(\begin{array}{cc}
J_{l-m}\left(\alpha_{R}\right) \Sigma_{R, 11}^{r, a,<}\left(\epsilon_{l}^{0}\right) J_{l-n}\left(\alpha_{R}\right) & J_{l-m}\left(\alpha_{R}\right) \Sigma_{R, 12}^{r, a,<}\left(\epsilon_{l}^{0}\right) J_{n-l}\left(\alpha_{R}\right) \\
J_{m-l}\left(\alpha_{R}\right) \Sigma_{R, 21}^{r, a,<}\left(\epsilon_{l}^{0}\right) J_{l-n}\left(\alpha_{R}\right) & J_{m-l}\left(\alpha_{R}\right) \Sigma_{R, 22}^{r, a,<}\left(\epsilon_{l}^{0}\right) J_{n-l}\left(\alpha_{R}\right)
\end{array}\right), \\
& \Sigma_{L, m n}^{r, a,<}(\epsilon)=\sum_{l}\left(\begin{array}{cc}
J_{l-m}\left(\alpha_{L}\right) \Sigma_{L, 11}^{r, a,<}\left(\epsilon_{l}^{-}\right) J_{l-n}\left(\alpha_{L}\right) & J_{l-m-N}\left(\alpha_{L}\right) \Sigma_{L, 12}^{r, a,<}\left(\epsilon_{l}^{+}\right) J_{n-l}\left(\alpha_{L}\right) \\
J_{m-l-N}\left(\alpha_{L}\right) \Sigma_{L, 21}^{r, a,<}\left(\epsilon_{l}^{-}\right) J_{l-n}\left(\alpha_{L}\right) & J_{m-l}\left(\alpha_{L}\right) \sum_{L, 22}^{r, a,<}\left(\epsilon_{l}^{+}\right) J_{n-l}\left(\alpha_{L}\right)
\end{array}\right),
\end{aligned}
$$

in which $\alpha_{\beta} \equiv \frac{W_{\beta}}{\omega}$ is the MW strength on the $\beta$ th tunnel barrier, $J_{n}(x)$ the $n$th Bessel function, $\epsilon_{l}^{0} \equiv \epsilon-l \omega$ and $\epsilon_{l}^{ \pm} \equiv \epsilon-l \omega \pm V_{N}, V_{L}=V_{N}$ and $V_{R}=0$ are set as a convention. Correspondingly, the equations for $G^{r}, G^{a}$, and $G^{<}$are Fourier transformed into $\mathbf{G}^{r, a}(\epsilon)=$ $\mathbf{g}^{r, a}(\epsilon)+\mathbf{g}^{r, a}(\epsilon) \boldsymbol{\Sigma}^{r, a}(\epsilon) \mathbf{G}^{r, a}(\epsilon)$ and $\mathbf{G}^{<}(\epsilon)=\mathbf{G}^{r}(\epsilon) \boldsymbol{\Sigma}^{<}(\epsilon) \mathbf{G}^{a}(\epsilon)$, or equivalently,

$$
\begin{aligned}
\mathbf{G}^{r, a}(\epsilon) & =\mathbf{g}^{r, a}(\epsilon)+\mathbf{g}^{r, a}(\epsilon) \boldsymbol{\Sigma}^{r, a}(\epsilon) \mathbf{g}^{r, a}(\epsilon)+\mathbf{g}^{r, a}(\epsilon) \boldsymbol{\Sigma}^{r, a}(\epsilon) \mathbf{g}^{r, a}(\epsilon) \boldsymbol{\Sigma}^{r, a}(\epsilon) \mathbf{g}^{r, a}(\epsilon)+\cdots, \\
\mathbf{G}^{<}(\epsilon) & =\left[\mathbf{g}^{r}(\epsilon)+\mathbf{g}^{r}(\epsilon) \boldsymbol{\Sigma}^{r}(\epsilon) \mathbf{g}^{r}(\epsilon)+\cdots\right] \boldsymbol{\Sigma}^{<}(\epsilon)\left[\mathbf{g}^{a}(\epsilon)+\mathbf{g}^{a}(\epsilon) \boldsymbol{\Sigma}^{a}(\epsilon) \mathbf{g}^{a}(\epsilon)+\cdots\right] .
\end{aligned}
$$

We note that finite order perturbation expansion is inadequate in the problem, because the formation of PAAS involves up to infinite order of tunneling processes. To re-sum up the series, we adopt the resonant approximation [12]

$$
\frac{1}{\left(\epsilon-l_{1} \omega \pm E_{0}+\mathrm{i} 0^{+}\right)} \cdot \frac{1}{\left(\epsilon-l_{2} \omega \pm E_{0}+\mathrm{i} 0^{+}\right)} \approx \delta_{l_{1} l_{2}} \frac{1}{\left(\epsilon-l_{1} \omega \pm E_{0}+\mathrm{i} 0^{+}\right)^{2}} .
$$

The approximation implies that the overlap between sidebands $E_{0}+l_{1} \omega$ and $E_{0}+l_{2} \omega$ can be ignored if $l_{1} \neq l_{2}$, which is justified when $\Gamma_{\beta} \ll \omega$. Applying the approximation to Eq.(17) and Eq.(18), one can obtain the solution

$$
\mathbf{G}_{m n}^{r, a,<}(\epsilon)=\left(\begin{array}{cc}
\delta_{m n} \sum_{k} \widehat{G}_{m k, 11}^{r, a,<} & \widehat{G}_{m n, 12}^{r, a,<} \\
\widehat{G}_{n m, 21}^{r, a,<} & \delta_{m n} \sum_{k} \widehat{G}_{k n, 22}^{r, a,<}
\end{array}\right),
$$

in which

$$
\begin{aligned}
& \widehat{G}_{m n}^{r, a}=\left[\left(\widehat{g}_{m n}^{r, a}\right)^{-1}-\widehat{\Sigma}_{m n}^{r, a}\right]^{-1}, \\
& \widehat{G}_{m n}^{<}=\widehat{G}_{m n}^{r} \widehat{\Sigma}_{m n}^{<} \widehat{G}_{m n}^{a},
\end{aligned}
$$

with

$$
\begin{gathered}
\widehat{g}_{m n}^{r, a}=\left(\begin{array}{cc}
\mathbf{g}_{m m, 11}^{r, a}(\epsilon) & 0 \\
0 & \mathbf{g}_{m n, 22}^{r, a}(\epsilon)
\end{array}\right), \\
\widehat{\Sigma}_{m n}^{r, a,<}=\left(\begin{array}{cc}
\boldsymbol{\Sigma}_{m m, 11}^{r, a,<}(\epsilon) & \boldsymbol{\Sigma}_{m n, 12}^{r, a,<}(\epsilon) \\
\boldsymbol{\Sigma}_{n m, 21}^{r, a,<}(\epsilon) & \boldsymbol{\Sigma}_{n n, 22}^{r, a,<}(\epsilon)
\end{array}\right) .
\end{gathered}
$$


It can be shown that the relations $\mathbf{G}^{r}=\left(\mathbf{G}^{a}\right)^{\dagger}$ and $\mathbf{G}^{<}=-\left(\mathbf{G}^{<}\right)^{\dagger}$ still hold in the solution.

With these Green functions, the time-dependent current can be expressed in a summation over ac components

$$
I_{\beta}(t)=2 \operatorname{Re} \sum_{l} e^{\mathrm{i} l \omega t}\left\{\int \frac{d \epsilon}{2 \pi} \operatorname{Tr} \sigma_{z}\left[\mathbf{G}^{r}(\epsilon) \boldsymbol{\Sigma}_{\beta}^{<}(\epsilon)+\mathbf{G}^{<}(\epsilon) \boldsymbol{\Sigma}_{\beta}^{a}(\epsilon)\right]_{l 0}\right\} .
$$

The current formula can be applied to the study of ac harmonics. However, we are more interested in the dc component $\bar{I}=\bar{I}_{L}=-\bar{I}_{R}$, due to the experimental reasons. To produce analytical results, we take the limit $\Delta \rightarrow \infty$, due to which all single particle processes are forbidden and Andreev reflection is the only conducting mechanism. After some algebra and take the limit $\eta \rightarrow 0$ (notice that $\lim _{\eta \rightarrow 0} \frac{\eta}{x^{2}+\eta^{2}}=\pi \delta(x)$ ), one can derive

$$
\begin{aligned}
\bar{I}= & 2 \sin \phi \frac{\Gamma_{L} \Gamma_{R}}{4}(-) \sum_{m} J_{m-N}\left(2 \alpha_{L}\right) J_{m}\left(2 \alpha_{R}\right) \frac{1}{\left(E_{m}^{+}-E_{m}^{-}\right)^{2}}\left[F\left(E_{m}^{+}\right)+F\left(E_{m}^{-}\right)\right], \\
F(\epsilon)= & \left(\epsilon+E_{0}-\frac{1}{2} m \omega\right) \sum_{l}\left[f\left(\epsilon_{l}^{0}+\frac{1}{2} m \omega\right) J_{l}^{2}\left(\alpha_{R}\right)+f\left(\epsilon_{l}^{-}+\frac{1}{2} m \omega\right) J_{l}^{2}\left(\alpha_{L}\right)\right]+ \\
& \left(\epsilon-E_{0}+\frac{1}{2} m \omega\right) \sum_{l}\left[f\left(\epsilon_{l}^{0}-\frac{1}{2} m \omega\right) J_{-l}^{2}\left(\alpha_{R}\right)+f\left(\epsilon_{l}^{+}-\frac{1}{2} m \omega\right) J_{-l}^{2}\left(\alpha_{L}\right)\right] .
\end{aligned}
$$

in which $E_{m}^{ \pm}= \pm \sqrt{\left(E_{0}-\frac{1}{2} m \omega\right)^{2}+\left|\Gamma_{m}\right|^{2}}$ and $\Gamma_{m}=\frac{1}{2}\left[\Gamma_{R} J_{m}\left(2 \alpha_{R}\right)+\Gamma_{L} e^{-i \phi} J_{m-N}\left(2 \alpha_{L}\right)\right]$. Eq.(26) is the central result of this paper, which is for the dc component of Josephson current in the resonant tunneling through mesoscopic superconducting junctions.

Before numerical study, we make a few remarks on this result: (1) The phase dependence of $\bar{I}$ is mainly determined by the prefactor $\sin \phi$, and a weak $\cos \phi$ dependence is hidden in $E_{m}^{ \pm}$via $\Gamma_{m}$. For this reason, $\phi$ is set as $\frac{\pi}{2}$ in the numerical calculation. (2) For the special case $N=0, \alpha_{L}=\alpha_{R}=0$, and $\Gamma_{L}=\Gamma_{R}=\Gamma, \bar{I}=2 \sin \phi \frac{\Gamma^{2}}{4} \frac{1}{\tilde{E}_{0}}\left[f\left(-\tilde{E}_{0}\right)-f\left(+\tilde{E}_{0}\right)\right]$, with $\tilde{E}_{0}=\sqrt{E_{0}^{2}+\left(\Gamma \cos \frac{\phi}{2}\right)^{2}}$, which reproduces the exact result for dc Josephson current through a resonant level. (3) The current formula is for the gauge choice of $V_{L}=V_{N}$ and $V_{R}=0$. It is easy to see that the formula is invariant under the transformation $L \longleftrightarrow R$, $V_{N} \rightarrow-V_{N}, N \rightarrow-N$, and $E_{0} \rightarrow E_{0}-V_{N}$, which corresponds to the gauge choice $V_{L}=0$ and $V_{R}=-V_{N}$. (4) $E_{m}^{ \pm}$are the poles of $\mathbf{G}^{r}(\epsilon)$, which can be interpreted as PAAS. Obviously, $E_{m}^{ \pm}$ contribute to the supercurrent with opposite signs. One can expect that resonant structures will appear near $E_{0}=\frac{1}{2} m \omega$. (5) Bessel functions enter the current formula not in the square form $J_{n}^{2}(x)$. It can be shown that $\bar{I}\left(\alpha_{L}, \alpha_{R}, \phi\right)=\bar{I}\left(-\alpha_{L},-\alpha_{R}, \phi\right)$ for $N$ even and $\bar{I}\left(\alpha_{L}, \alpha_{R}, \phi\right)=\bar{I}\left(-\alpha_{L},-\alpha_{R}, \phi+\pi\right)$ for $N$ odd 
numerical results-Firstly, we discuss the case that MW is applied symmetrically to the left and right tunnel barrier, i.e., $\alpha_{L}=\alpha_{R}$. Fig.2 shows the curves of $I_{c}=\bar{I}\left(\phi=\frac{\pi}{2}\right)$ vs $E_{0}-\frac{V_{N}}{2}$ at bias voltage $V_{N}=\frac{N}{2} \omega$, with $N=0,1,2,3,4$ (the curves are shifted by $\frac{V_{N}}{2}$ for demonstration). Four features are noteworthy in the plot: (1) The curve is symmetric (anti-symmetric) with respect to $E_{0}=\frac{V_{L}+V_{R}}{2}=\frac{V_{N}}{2}$ for $N$ even (odd). (2) Photon-assisted structures appear near $E_{0}=V_{L}-\frac{1}{2}(N-m) \omega=V_{R}+\frac{1}{2} m \omega$. (3) There are two types of resonant structures, single peak (dip) and peak-dip pair. (4) The structures grow with the MW strength non-monotonously.. Feature (1) is due to the relation $I\left(E_{0}, \phi\right)=I\left(V_{N}-E_{0}, \phi\right)$ for $N$ even and $I\left(E_{0}, \phi\right)=I\left(V_{N}-E_{0}, \phi+\pi\right)$ for $N$ odd. Using $I\left(E_{0},-\phi\right)=-I\left(E_{0}, \phi\right)$, one can obtain $I\left(E_{0}, \phi=\frac{\pi}{2}\right)=(-)^{N} I\left(V_{N}-E_{0}, \phi=\frac{\pi}{2}\right)$. Feature (2) can be understood in terms of PAAS shown in Fig.1b and 1c: Electron and hole are Andreev reflected back and forth by the superconducting gaps. When $E_{0}$ is near the position $V_{L}-\frac{1}{2}(N-m) \omega=V_{R}+\frac{1}{2} m \omega$, quasiparticles may exchange $N-m$ photons with the MW field at the left tunnel barrier and exchange $m$ photons at the right, so that the trajectory is closed and bound states are formed. It is these PAAS that play the role of carrying supercurrent. Understanding of feature (3) and (4) needs quantitative analysis of each photon-assisted structure. To proceed, we decompose the total current into $\bar{I}=\sum_{m} I_{m}$, and expand $I_{m}$ near $E_{0}=\frac{1}{2} m \omega$. Let $E_{0}=\frac{1}{2} m \omega+\delta$, one can obtain $I_{m}=I_{m}^{+}+I_{m}^{-}$with

$$
\begin{aligned}
I_{m}^{ \pm}= & 2 \sin \phi \frac{\Gamma^{2}}{4}(-) J_{m-N}\left(2 \alpha_{L}\right) J_{m}\left(2 \alpha_{R}\right) \frac{1}{4\left(\delta^{2}+\left|\Gamma_{m}\right|^{2}\right)} \\
& {\left[\alpha_{m}^{ \pm}\left( \pm \sqrt{\delta^{2}+\left|\Gamma_{m}\right|^{2}}+\delta\right)+\beta_{m}^{ \pm}\left( \pm \sqrt{\delta^{2}+\left|\Gamma_{m}\right|^{2}}-\delta\right)\right] . }
\end{aligned}
$$

The cancelation between $I_{m}^{+}$and $I_{m}^{-}$results in two types of resonant structures: $C_{1} \frac{1}{\sqrt{\delta^{2}+\left|\Gamma_{m}\right|^{2}}}+$ $C_{2} \frac{\delta}{\delta^{2}+\left|\Gamma_{m}\right|^{2}}$, corresponding to single peak (dip) and peak-dip pair. At zero temperature, $C_{1}$ and $C_{2}$ are proportional to

$$
\begin{aligned}
C_{1} & \propto \sum_{l}\left[\delta_{l, \frac{1}{2} m} J_{l}^{2}\left(\alpha_{R}\right)+\delta_{l, \frac{1}{2}(m-N)} J_{l}^{2}\left(\alpha_{L}\right)\right], \\
C_{2} & \propto\left[\sum_{l>-\frac{1}{2} m} J_{l}^{2}\left(\alpha_{R}\right)-\sum_{l>\frac{1}{2} m} J_{l}^{2}\left(\alpha_{R}\right)+\sum_{l>-\frac{1}{2}(m-N)} J_{l}^{2}\left(\alpha_{L}\right)-\sum_{l>\frac{1}{2}(m-N)} J_{l}^{2}\left(\alpha_{L}\right)\right],
\end{aligned}
$$

The oscillatory nature of Bessel functions in these coefficients is responsible for feature (4). There is an interesting special case, $N=2, m=1, \alpha_{L}=\alpha_{R}$, due to which $C_{1}=C_{2}=0$ for arbitrary MW strengths. Correspondingly, the resonant structure near $E_{0}-\frac{V_{N}}{2}=0$ is always missing for $N=2$. 
Next, we investigate the case that MW is applied only to one of the tunnel barriers, i.e., $\alpha_{L}=0$ and $\alpha_{R} \neq 0$. Fig.3 shows the curves of $I_{c}$ vs $E_{0}$ for $\alpha_{L}=0$ and $\alpha_{R}=1$. In contrast to the symmetric case, a single peak is pinned at $E_{0}=V_{L}$. The reason is as follows: since no MW is applied to the left barrier, Andreev tunneling through this barrier occurs only when $E_{0}$ lines up with $V_{L}$, while photon-assisted processes is allowed at the right barrier irradiated by MW field. The inset shows $I_{c}$ vs MW strength $\alpha_{R}$ at $E_{0}=V_{L}$ for $N>0$ (The case of $N<0$ can be easily deduced from $N>0$ ). One can see in the plot that the peak height (including the sign) is predominated by the prefactor $J_{m-N}\left(2 \alpha_{L}\right) J_{m}\left(2 \alpha_{R}\right)$. For $\alpha_{L}=0, J_{m-N}\left(2 \alpha_{L}\right)$ requires $m=N$, and the peak height is proportionate to $J_{N}\left(2 \alpha_{R}\right)$. We note that there exist some regions of MW strength where $I_{c}$ and $V_{N}$ have opposite signs, which can be viewed as quantum pump effect. However, this feature is dramatically different from the quantum pump effect in normal mesoscopic junctions.

conclusion-To sum up, we have investigated ac Josephson effect in the resonant tunneling through mesoscopic superconducting junctions. We show that PAAS play an essential role for conducting supercurrent through a narrow resonance, in which MAR trajectory is closed by exchanging photons with MW field. On the Shapiro steps $2 V=N \omega$, de component appears when the resonant level $E_{0}$ is near $V_{L}-(N-m) \omega=V_{R}+m \omega$, due to the formation of PAAS. In the limit $\Delta \rightarrow \infty$, analytical result, Eq.(26), is derived for the de component of Josephson current, and help to understand new features of ac Josephson effect in the case of resonant tunneling. We have dropped Coulomb interaction in the calculation, the derived results are meaningful in the following senses: (1) They are directly applicable to the systems where Coulomb blockade effect is negligible, i.e., $U \sim \Gamma \ll \Delta$ ( $U$ is the charging energy). This is possible by using substrate with large dielectric constant to reduce $U$ or using proper material as S lead to obtain large $\Delta$. (2) They are instructive for more complicated cases. We note that the conception of PAAS is also useful for the interacting case. For $U \gg \Gamma$, the resonant level $E_{0}$ is effectively split into two resonances $E_{0}$ and $E_{0}+U$. Similar to Fig.1b and 1c, one can draw diagrams of closed MAR trajectory through these resonances. Moreover, Coulomb blockade can be partially removed by bias voltage or MW irradiation, as long as $U$ is comparable to $\Delta$. Obviously, calculation including interaction term is much more difficult, and analytical results are almost impossible. Efforts along this line are still in progress.

This project was supported by NSFC under Grants No. 10074001 and No. 90103027, and also by the support from the Visiting Scholar Foundation of State Key Laboratory for 
Mesoscopic Physics in Peking University.

* To whom correspondence should be addressed. 


\section{REFERENCES}

[1] C. J. Muller, J. M. van Ruitenbeek, and L. J. de Jongh, Phys. Rev. Lett. 69, 140 (1992).

[2] N. van der Post, et al., Phys. Rev. Lett. 73, 2611 (1994); B. Ludoph et al., Phys. Rev. B 61, 8561 (2000).

[3] E. Scheer et al., Phys. Rev. Lett. 78, 3535 (1997); E. Scheer et al., Nature 394, 154 (1998).

[4] M. F. Goffman et al., Phys. Rev. Lett. 85, 170 (2000); R. Cron et al., Phys. Rev. Lett. 86, 4104 (2001).

[5] D. C. Ralph, C. T. Black, and M. Tinkham, Phys. Rev. Lett. 74, 3241 (1995).

[6] N. Knorr et al., Phys. Rev. Lett. 88, 096804 (2002).

[7] M. R. Buitelaar, T. Nussbaumer, and C. Schönenberger, Phys. Rev. Lett. 89, 256801 (2002).

[8] H. Takayanagi, T. Akazaki, and J. Nitta, Phys. Rev. Lett. 75, 3533 (1995).

[9] A. L. Yeyati et al., Phys. Rev. B 55, R6137 (1997).

[10] G. Johansson et al., Physica C 293, 77 (1997), Phys. Rev. B 60, 1382 (1999).

[11] J. C. Cuevas et al., Phys. Rev. Lett. 88, 157001 (2002).

[12] Q. -f. Sun, J. Wang, and T. -h. Lin, Phys. Rev. B 59, 13126 (1999).

\section{FIGURE CAPTIONS}

Fig. 1 Schematic diagram of the resonant tunneling through mesoscopic superconducting junctions. (a): Without MW irradiation, MAR trajectory can not be covered by a narrow resonance, and the tunnel current is exponentially small. (b) and (c): In the presence of MW irradiation, MAR trajectory can be closed by emitting or absorbing photons. Two types of PAAS are formed, carrying supercurrent with opposite signs.

Fig. 2 The curves of $I_{c} \equiv \bar{I}\left(\phi=\frac{\pi}{2}\right)$ vs $E_{0}-\frac{V_{N}}{2}$ at bias voltage $V_{N}=\frac{N}{2} \omega$ ( $N$ from 0 to 4$)$, with symmetric MW strengths $\alpha_{L}=\alpha_{R}$. Parameters are: $\omega=1, \Gamma_{L}=\Gamma_{R}=0.02$, $k_{B} T=0.001 . V_{L}=V_{N}$ and $V_{R}=0$ are set as a convention. 
Fig. 3 The curves of $I_{c} \equiv \bar{I}\left(\phi=\frac{\pi}{2}\right)$ vs $E_{0}$ at bias voltage $V_{N}=\frac{N}{2} \omega(N$ from -4 to 4$)$, with MW strengths $\alpha_{L}=0$ and $\alpha_{R}=1$. Other parameters are the same as Fig.2. The inset shows $I_{c}$ at $E_{0}=V_{L}$ vs the MW strength $\alpha_{R}$. Diagrams for corresponding PAAS is also shown in the plot. 